\title{
DAS GESPRÄCH ZWISCHEN HÖLDERLIN, HEGEL UND SCHELLING ÜBER KANTS ANTINOMIEN
}

\author{
Fernando Manuel Ferreira da Silva \\ (Centro de Filosofia da Universidade de Lisboa)
}

Aufgrund des erbarmungslosen Einflusses der Zeit, handelt es sich leider um einen sehr lückenhaften Briefwechsel, der die drei jungen Philosophen Hegel, Hölderlin und Schelling als gleichzeitige Hauptfiguren hat - nur zehn Briefe, von Dez. 1794 bis Juni 1796, sind uns übermittelt worden. Sie entsprechen einem langen Zeitraum geistiger Reife für die drei Freunde, wie auch einer Trennung von ungefähr anderthalb Jahren zwischen Hölderlin und Hegel, die vor über einem Jahr den Tübinger Stift verlassen hatten, und Schelling, der dort bis zum Herbst 95 bleiben würde; und verkörpern deswegen den allerletzten Hauch einer tapferen Generation, die, trotz des orthodoxen Zwangs der strengen Tübinger Regierung, doch gierig und treuherzig solche gewaltigen (und nicht minder subversiven) Triebfedern wie Jacobis Romane, die Französische Revolution oder Schillers Ideal einer ästhetischen Erziehung einnahm.

Unter all diesen Triebfedern bringt aber der Briefwechsel zwischen den drei Freunden eine andere vor, die bestimmt die wichtigste und entscheidendste für die geistige Entwicklung der drei Studenten war: die der Kantischen Kritik der supernaturalistischen Orthodoxie Storrs oder Flatts, nach welchem Kant, indem er die Autonomie der Vernunft über die dogmatische Autorität des Glaubens den Vorrang gibt, auch die Notwendigkeit der rationalen Begrenzung des moralischen Gefühls, der Erfahrung Gottes und selbst der Lehr- und Lernübungen der Religion behauptet, die Möglichkeit einer Erkenntnis ausserhalb der Erfahrung streitet und, folglich, die orthodoxen Glaubensartikeln durch den Begriff der Zweckmässigkeit als bloße biblische Buchstabe verneint. Sich selbst als Kantianer innerhalb dieses Problem betrachtend, wussten die drei jungen Freunden auch, dass die gegenwärtige Frage nicht ein bloßes 
Problem der Begrenzung zwischen Philosophie und Religion war. Dies war vielmehr der erste Schritt zur Wahrnehmung, dass die Begriffe selbst von Philosophie und Religion nicht mehr anders erreicht oder gedacht werden könnten, als durch die Absonderung ihrer spezifischen Beweisarten, und dass ein solches Problem, durch Kant erwacht, nicht nur das Hauptproblem der damaligen Philosophie, sondern auch das echte Triebwerk der unendlichen Mannigfaltigkeit des deutschen Idealismus war. Ich deute natürlich auf den allmählichen annähernden Feilensprozess der Philosophie durch das philosophische Wort selbst, der sich als einen entscheidenden Beitrag zur allmählichen Positionierung der Philosophie im Gebiet des Wissens so wie zu ihre Szientifizierung im Bereich der menschlichen Erkenntnis erweisen würde; ein Prozess wo, nach Fichte selbst, Begriff und Sprache unzertrennlich, unfehlbar und allgemeingültig sein sollten, damit eine solche Aufgabe ausgeführt werden könnte.

Die Stellung der drei Freunde gegenüber dieser Frage ist also so einstimmig wie eindeutig sichtbar. Von Schelling, dem best positionierten, um die damalige Lage zu kommentieren, wissen wir dass nach dem ersten geistigen Aufruhr, der Anfang 1795 durch die kantische Schriften veranlasst war, «d $\mathrm{d}$ as] alte Unkraut» $(B, 1: 13)$ noch einmal gewachsen war, dass «der philos. Geist (...) hier bereits seinen Meridian erreicht» (id.) hatte und selbst, dass «nach vieler Mühe nun endlich unsre Philosophen den Punkt gefunden [haben], wie weit man (da es nun einmal ohne die leidige Philosophie nimmer fort will) mit dieser Wissenschaft gehen dürfe». (id.) «Ich bin fest überzeugt, dass der alte Aberglaube (...) in den Köpfen der meisten schon wieder mit den kantischen Buchstaben combiniert ist» $(B, 1: 14)$. Öfters in seinen frühsten Schriften deutet auch Hölderlin auf dieselbe Verachtung gegen die Orthodoxie im Stift, und selbst Hegel behauptete schon in Dez. 94, dass «Ehe nicht eine Art von Reinhold oder Fichte dort auf einem Katheder sitzt, nichts Reelles herauskommen [wird]. Nirgends wird wohl so getreulich wie dort das alte System fortgepflanzt.» $(B, 1: 12)$

Eben infolge dieser Wörter und innerhalb der Problemlage Tübingens liegt die Frage, die ich Ihnen heute vorbringe. Sie entspricht dem vollendetsten Produkt dieser Problemrahmung, wie auch dem ersten Augenblick, in dem die Stimmen der drei Freunde sich zum ersten Mal vereinigen. Er kommt zweifach vor; erstens in Hegel, als er Ende Januar Schellings Brief so beantwortet:

«Zu dem Unfug, wovon du schreibst und dessen Schlussart ich mir danach vorstellen kann, hat aber unstreitig Fichte durch seine Kritik aller Offenbarung Tür und Angel geöffnet. (...) Wenn seine Grundsätze einmal fest angenommen sind, so ist der theologischen 
Logik kein Ziel und Damm mehr zu setzen. Er räsoniert aus der Heiligkeit Gottes, was er vermöge seiner rein moralischen Natur tun müsse u.s.w., und hat dadurch die alte Manier, in der Dogmatik zu beweisen, wieder eingeführt (...). Wenn ich Zeit hätte, so würde ich suchen, es näher zu bestimmen, wieweit wir - nach Befestigung des moralischen Glaubens die legitimierte Idee von Gott jetzt rückwärts brauchen z. B. in Erklärung der Zweckbeziehung u.s.w., sie von der Ethikotheologie her jetzt zur Physikotheologie mitnehmen (...) dürften. Dies scheint mir der Gang überhaupt zu sein, den man bei der Idee der Vorsehung ( ...) nimmt u.s.w.» $(B, 1: 17)$

Zweitens, in dem darauffolgenden Brief, der berühmte Brief, den Hölderlin an Hegel am 26. 1.95 schrieb, wo er seine erste Stellung gegen Fichte einnahm, vor allem aber, wo er sich freut, dass Hegel sich «an die Religionsbegriffe mach[t]». Die Stelle lautet:

«Den Begriff der Vorsehung behandelst du wohl ganz parallel mit Kants Teleologie; die Art, wie er den Mechanismus der Natur (also auch des Schicksals) mit ihrer Zweckmässigkeit vereiniget, scheint mir eigentlich den ganzen Geist seines Systems zu enthalten; es ist freilich dieselbe, womit er alle Antinomien schlichtet.» $(B, 1: 20)$

Verschiedene Fragen, einige sich selbst einleuchtend, andere sich selbst verbergend, sind von diesen beiden Zeugnissen aufgestellt. Die klarste ist aber, dass die unmittelbarste Fortführung dieses Problems für die drei Freunde in Fichte lag, doch so, dass durch Fichte selbst die unerwartete Wiederherstellung des dogmatischen «Unkraut[s]» in Tübingen veranlasst wurde. Denn wenn die Lösung, wie bei Kant, in der Unzertrennbarkeit zwischen der Vorsehung (dem Schicksal) und die Zweckmässigkeit - sowie zwischen Subjekt und Objekt - bestand (d.h., die Antinomien, die Klüfte, die unüberwindbaren Gegenteile zwischen Mensch und Natur nicht zu befördern), sondern sie zu schlichten oder, wenn möglich, aufzuheben zu versuchen, so könnte das nur zwei Folgen haben: erstens, dass trotz Fichtes kühnem Versuch die Antinomien aufzuheben, ein solcher Versuch den drei Freunden als unzulänglich vorkommen musste; und zweitens, wenn das Problem in der Form, in der lebendigsten Tätigkeit und deswegen in dem eigentlichen Kern der Philosophie lag, so setzte die Suche auf die Zurückführung der Frage bei Kant immer voraus, dass die drei Studenten das, was in Fichtes Theorie gegenüber Kants abwich, umzukehren gezwungen waren.

Lassen wir uns nun mit Hegels Position anfangen. Hegel oblag, um Hölderlin zu paraphrasieren, sich mit dem theologischen Teil des Problems zu beschäftigen. Unter den fragmentarischen Texten, die Hegel bis Juni 94 verfasste, alle von theologischem Inhalt, zeichnet sich ein 
zentraler Text aus, vielleicht der allerletzte, den er in Tübingen (im Sommer 93) schreiben würde, mit dem Titel «Religion ist eine der wichtigsten Angelegenheiten...» (GW, 1: 83-114). Im Text gründet Hegel die schon erwähnte in den Ursprung seines philosophischen Werdegangs so entscheidende Distinktion zwischen Ethikotheologie - die objektive Religion, die Religion des Verstandes und des Gedächtnisses, des «Kabinett[s] des Naturlehrers» $(G W, 1: 88)$, der allgemeinen Theologie, dessen hermeneutische Kunstgriffe sich nur nach der Systematisierung der Religion und dem Bild Gottes als Grundlage desselben System erstreben - und einer anderen Art von Religion, die Physikotheologie, die subjektive, lebendige und sinnliche Religion, die Hegel beisteht, in dem nicht der Verstand herrscht, sondern die «(...) Sinnlichkeit das HauptElement bei allem Handeln und Streben der Menschen ist (...)» ( $G W, 1: 84)$, und die Religion, die selbst sinnlich ist, aus sinnlichen Menschen, deren «(...) religiöse Triebfedern zum Guthandeln sinnlich seyn [müssen], um auf die Sinnlichkeit wirken zu können» $(G W, 1: 86)$, besteht. Eine, sagt Hegel, die uns in der Schule durch tote Formeln gegeben ist, nach welcher wir als Menschen, unmittelbare und deswegen passive Empfänger von Gottes übertragenem Wort, Eigentümlichkeiten und Glaubensartikeln (wie die Vorsehung) sind, die uns nur positiv beeinflussen können; die andere, die Religion der Sinnlichkeit, nach welcher laut Hegel «in einem System der Moral reine Moralität von Sinnlichkeit in Abstrakto gesondert werden muss, so sehr diese unter jene erniedrigt wird (...)» $(G W, 1: 84)$, die sich aber «(...) auf alle Zweige der menschlichen Neigungen (ohn dass die Seele gerade es sich bewust ist,) [verbreitet] und wirkt überall - aber nur mittelbar mit - sie wirkt, um mich so auszudrükken, negativ (...)» $(G W, 1$ : 90).

Die hier angewendeten Begriffe sind also dieselben die Hegel schon in dem Brief an Schelling benutzt, dieselben die Hölderlin auch schon kannte, und nur ein Ziel haben: in beiden Fällen unterstreicht Hegel die unbedingte Notwendigkeit, von der objektiven zur subjektiven Religion überzugehen, denn jene ist nur ein «Bestandteil» (id.) dieser, und ehe diese jene verkörpert, würde die philosophische/theologische Beweisart immer die der dogmatischen Logik bleiben. Was bedeutet das? Es bedeutet, dass die richtige Beweisart nicht aus der Idee von Gott ausgehen kann, wie bei Fichte, sondern, dass diese eben von dem Menschen ausgehen muss; was wiederum bedeutet, dass Hegels Gott absolut subjektiv ist und wirkt, aber nicht, dass man nach ihm und dieser Subjektivität halber fortrücken muss. Die Subjektivität, erklärt Hegel, ist unsere eigene Subjektivität, und die Religion ist deswegen nicht das Produkt, sondern der Prozess einer Beweisart, die jetzt umkehrend subjektiv ist und auf Gott durch ein lebendiges (und nicht einseitiges) Gespräch zwischen Objekt und Subjekt gehen soll. 
Die Frage bestand also zuerst in der Erreichung einer Vereinigungssphäre, die dieses Gespräch selbst befördern, und so die friedliche aber auch wirkliche (und deswegen widerstandsfähige) Koexistenz zwischen Objekt und Subjekt, um die Antinomien zu schlichten, ermöglichen könnte. Dann - und nur dann - könnte die Umänderung oder sogar vollständige Umkehrung der Positionen dieser beiden, Mensch und Gott, ohne sie zu lädieren, stattfinden. Anders gesagt, die Lösung bestände immer in der Triebfeder dieses umkehrenden Prozesses: nach Hegel, «die Erklärung der Zweckbeziehung» $(B, 1: 17)$.

Hegels Problem, denke ich, wird noch deutlicher, als er kurz danach und fast gleichzeitig zwei Briefe empfängt, ein Brief von Hölderlin, der andere von Schelling. Beide analysieren dieses Problem auf einer offenbar widersprechenden Art. Es war aber Hegel selbst, der die entgegengesetzte Meinungen seiner Freunde veranlasste, indem er Schelling gesteht, eine Stelle in seinen Philosophischen Briefen über Dogmatismus und Kritizismus nicht verstanden zu haben:

«Einen Ausdruck in Deinem Briefe von dem moralischen Beweise verstehe ich nicht ganz: «den sie so zu handhaben wissen, dass das individuelle, persönliche Wesen herausspringe.» Glaubst $\mathrm{Du}$, wir reichen eigentlich nicht so weit?» $(B, 1: 18)$.

In dem folgenden Brief (nachdem Hegel Hölderlins erwähnte Brief empfangen hatte), scheut sich Schelling nicht, mit Hegel einverstanden zu sein, dass «solange jene Schlussart, die Fichte in der Kritik aller Offenbarung (...) wieder aufbrachte, noch als gültig angesehen wird, der philosophischen Torheit kein Ende ist.» ( $B, 1: 21)$ Als Antwort auf Hegels Frage aber (indem er wusste, dass die Frage schon längst bei Hegel und Hölderlin entschieden war $(B, 1: 22))$, behauptet Schelling nicht, dass das persönliche Wesen unerreichbar ist, sondern dass es möglich ist, darüber hinauszugehen. Auch für Schelling «[waren] die orthodoxen Begriffe von Gott nicht mehr». (id.) Schelling war indessen also Spinozist geworden, indem das absolute Objekt für Spinoza alles gegenüber dem Subjekt (als Gipfel der dogmatischen Philosophie) war, während für Schelling eben das absolute Ich alles gegenüber dem Objekt (als Gipfel der kritischen Philosophie) war. Wenn man aber die schellingsche Inversion näher betrachtet, merkt man, dass Schelling das Problem einer philosophischen und theologischen Beweisart ganz anders als Hegel und Hölderlin betrachtete. Denn es ist unleugbar, dass Schelling auf dieser Weise ein «Gegenstück» Spinozas versucht und dass dieses ein gewissermaßen sehr vorteilhafter Fortschritt gegen das Problem Fichtes sein könnte. Denn indem er auf dem Subjekt den Anfangspunkt und auf Gott den letzten Punkt für die moralische 
Deduktion setzt (was gemäss Hegels Begriff einer subjektiven Religion ist), scheint Schelling die Stellung der zwei leitenden Pole der Deduktion umzukehren - was sich Fichtes Theorie entgegensetzen würde, wenn nur innerhalb eines solchen Systems das Objekt als ein blosser Bestandteil, als beträchtliche Einheit, die die Vereinigung zwischen Subjekt und Objekt ermöglicht, betrachtet würde. Für Schelling aber, ganz im Gegenteil, ist das höchste Prinzip der ganzen Philosophie ein rein absolutes, d. h., nicht ein durch das Objekt bedingtes, also durch Freiheit gesetztes Ich; anders gesagt, das Subjekt, das Schelling der moralischen Deduktion beilegt, ist, in aller Wahrheit, ein absolutes Subjekt, dessen Gang zu Gott aus absoluter Freiheit besteht. Und eben dadurch, dass man in einer theoretischen Ansicht diesem Subjekt noch durch die verschiedenen Sphären des Objekts begrenzen kann - was das Unbedingte mit sich selbst in Widerspruch setzen würde -, ist es notwendig, sagt Schelling, dass diese Einschränkungen aufgehoben sind, dass man die Endlichkeit hinter sich lässt und endlich in Gottes übersinnliche Welt eintritt: «Diese [die praktische Philosophie] fordert also Zerstörung der Endlichkeit und führt uns dadurch in die übersinnliche Welt. (Was der theoretischen Vernunft unmöglich war, sintemal sie durch das Objekt geschwächt war, das tut die praktische Vernunft.)» (id.)

Was dies bedeutet, sowie für Schelling als auch für Hegel (obwohl in verschiedenen Sinnen und vornehmlich mit verschiedenen Folgen für deren geistige Entwicklung), ist das Verschwinden des Objekts als deduzierbare Einheit im ganzen Prozess. Aber während für Schelling dies die Lösung des Problems war, war sie für Hegel eben das unüberwindbare Hindernis zur Möglichkeit einer Sphäre, wo die Antinomien geschlichtet und wo Gott und der Mensch, dieses persönliche Wesen, durch ein subjektives Verhältnis verbunden sein könnten. Denn es ist offenbar, dass, in diesem Fall, Gott nur scheinbar der letzte Punkt der moralischen Deduktion bleiben könnte. Schelling aber setzte Hegel wiederum entgegen, dass wenn es das Ich war, das durch eine Beweisart in welchem es selbst absolut und das Objekt nur in seiner eigenen Nicht-Existenz innerhalb des Subjekts existiert, die unendliche Sphäre erzeugt hat, so, indem wir zu Gott gelangen, finden wir dass auch Gott unter der Form des absoluten Ichs existiert - denn Gott als Gott, d. h., das Alles der theoretischen Ansicht, ist jetzt in Schellings unbedingt praktischer Ansicht $=0$ : «Allein in dieser [übersinnlichen Welt] können wir nichts finden als unser absolutes Ich, denn nur dieses hat die unendliche Sphäre beschrieben. Es gibt keine übersinnliche Welt für uns als die des absoluten Ichs. - Gott ist nichts als das absolute Ich, das Ich, insofern es Alles Theoretische zernichtet hat, in der theoretischen Philosophie also = 0 ist.» (id.) Mithin ist Gott für Schelling nicht, wie für Hegel, das Ende 
eines umgekehrt annähernden, d.h., eigentlich subjektiven Prozesses; es ist subjektiv, aber nur insofern Gott nicht mehr Gott ist, sondern nur das absolute Ich als etwas prä-determiniertes, also als ewig erreichbarer Instanz existiert. Nur dieses Ich existiert für Schelling, und sogar auf einer so unveränderlichen, so unfehlbaren, so übermässig sinnlichen Weise, dass dieser Gott oder dieses absolute Ich nie der wirklich letzte Punkt der moralischen Deduktion sein kann, sondern ein unendlich abspannender, unendlich ausdehnender Punkt, dem wir uns je mehr nähern können, desto mehr, schliesst Schelling, wir unsere eigene Personalität aufheben und uns vom Objekt entziehen. Schelling deutet also auf eine immer unvollendete (weil ewig kreisläufige) Bewegung, einen unendlich fühlbaren Prozess: «Mithin gibt es keinen persönlichen Gott, und unser höchstes Bestreben ist die Zerstörung unserer Persönlichkeit, Übergang in die absolute Sphäre des Seins, der aber in Ewigkeit nicht möglich ist; - daher nur praktische Annäherung zum Absoluten, und daher - Unsterblichkeit.» (id.)

Wie stark Schelling Hegels Theorie beeinflusst hat, ist heute leicht erkennbar. Bevor er aber dieser Brief von Schelling bekam, empfing er, wie gesagt, einen anderen von Hölderlin, den obenerwähnten Brief vom 26.1.95. In diesem Brief behauptet Hölderlin, dass auch er, so wie Hegel, «(...) [Fichte] sehr im Verdacht des Dogmatismus [hatt']; er scheint,» sagt Hölderlin, «wenn ich mutmassen darf, auch wirklich auf dem Scheidewege gestanden zu sein, oder noch zu stehn - er möchte über das Faktum des Bewusstseins in der Theorie hinaus» ( $B, 1: 19)$. Anders gesagt, indem Fichte die Lösung des Problems über das Bewusstsein (in der Identität) findet, überschreitet auch er, nach Hölderlin - wie auch nach Hegel -, die theoretischen Schranken des Problems. Allein ist es hier schwer zu sagen, ob für Hölderlin Fichte mehr ein Dogmatiker schien, weil er das Problem auf ein praktisches Gebiet versetzte, oder wegen der Art wie er es machte; denn trotz der Acht die Hölderlin vor seinem Professor hatte, das Bewusstsein $\mathrm{zu}$ übertreten meinte für Hölderlin es selbst aufzuheben, und nicht beiseite zu legen oder ein anderes Element unterzuordnen. Dies ist der Grund warum, für den jungen Hölderlin von «Urteil und Sein» (TS: 7-8), Fichte auch (so wie Schelling) seine Zuflucht zu Spinoza nehmen musste, um die subjektive Identität zu kristallisieren; denn «sein absolutes Ich (= Spinozas Substanz) enthält alle Realität; es ist alles, u. ausser ihm ist nichts» (id.); d.h., ausser ihm findet keine Freiheit statt. Dies hatte für den jungen Hölderlin und Hegel zwei entscheidende Folgen: erstens, dass, wenn ein Objekt nicht existiert, das Subjekt allgemein ist und deswegen gibt es auch kein Bewusstsein als Pendulum zwischen beiden; hier ist die fichtesche Identität als etwas, das leicht verwechselbar aber notwendig verschieden von dem «Sein» (wie auch Hegel ihn versteht), als 
unverbrüchliche Verbindung zwischen Subjekt und Objekt, zu verstehen ist. Zweitens, dass, auf diese Weise, Fichtes «Ich bin Ich» nicht nur die entgegengesetzten Pole in der Sphäre der Erkenntnis nicht vereinigte, sondern sie gewaltsam trennte, indem er ihnen das Bewusstsein entnahm.

Hölderlin aber begann über dieses Grundproblem nicht erst in den ersten Monaten 1795 nachzudenken. Hegel, es ist jedem bewusst, wusste das auch; denn schon seit August 1794 war Hölderlin ein geschickter Leser der Wissenschaftslehre, anders als sein Freund. Im ersten uns bekannten Fragment dieser Zeit, «Über das Gesetz der Freiheit» [«Es giebt einen Naturzustand (TS: 3-4)], finden wir den ersten Schritt zum obenerwähnten Punkt seiner eigenen Theorie, dieselbe die mit Hegels Theorie so einmütig sein würde. Hier behauptet Hölderlin, dass das Gesetz der Freiheit - dasselbe, das er in Fichte und Schelling nicht erkennen könnte - «gebietet, ohne alle Rücksicht auf die Hülfe der Natur» [TS: 4); um zu gebieten aber, braucht die Freiheit eine Art von Widerstand von der Natur - «sonst würde es nicht gebieten» (id.). Das heisst: ohne eine Entgegensetzung (wie im Fall Schellings, wo das Objekt keinen Widerstand dem absoluten Subjekt anbietet), ohne etwas, das sie eigentlich als Freiheit zeigte, würde die Freiheit sich nie als solche vorstellen, denn sie hätte nichts zu überwinden, keine Nicht-Freiheit über die sie hinausgehen musste. Deswegen, sagt Hölderlin, «das erstemal, dass das Gesetz der Freiheit sich an uns äussert, erscheint es strafend» (id.), und wenn das der Fall nicht wäre, «[würde] die Moralität nicht aufhör[en], Moralität zu sein, sobald die Bestimmungsgründe in der Natur und nicht in der Freiheit liegen»(id.), wie bei Fichte, und die Legalität der Freiheit - d. h., ihre Wirksamkeit sowie die Legitimität unserer Wahrnehmung der Phänomene der Natur, unserer selbst und Gott wäre - «ein sehr unsicheres, nach Zeit und Umständen wandelbares Ding.» (id.) Deswegen sieht Hölderlin in dieser Art von Widerstand, in diesem Berührungspunkt zwischen dem Mechanismus der Natur und ihrer Zweckmässigkeit, nicht nur den Hauptgrund seiner theoretischen Verwandtschaft mit Hegel, sondern auch die Similarität zwischen ihren und Kants Theorien als die Antwort auf die Frage der philosophischen Beweisart.

Im Grunde müsste es sowohl für Hölderlin als auch für Hegel nicht eine Totalität, sondern eine Interdependenz oder vielmehr eine zwanglose (doch nicht ganz friedliche, weil strafende) Koexistenz zwischen den Gegenteilen geben, so dass die Freiheit wirklich sein könnte. Anders gesagt, man musste ein versöhnendes Gebiet denken, wo nicht nur die Antinomien sich schlichten würden (wie bei Kant), sondern auch wo die Antinomien als solche gültig wären, ohne deswegen eine durch die Totalisierung der anderen aufzuheben. Man bedürfte also, nach Hölderlin, «(...) eine Seite des empirischen Begehrungsvermögens, die Analogie 
dessen, was Natur heisst, die am auffallendsten ist, wo das Notwendige mit der Freiheit, das Bedingte mit dem Unbedingten, das Sinnliche mit dem Heiligen sich zu verbrüdern scheint, eine natürliche Unschuld, man möchte sagen eine Moralität des Instinkts, und die ihm gleichgestimmte Phantasie ist himmlisch.» (TS: 3 ).

Hier taucht aber das wahre Problem einer versöhnenden «Seite» zwischen Gegenteilen auf. Denn wenn der Mensch ewig abhängig von Naturursachen ist, sagt Hölderlin, so ist es ihm unmöglich dieses Gebiet $\mathrm{zu}$ fixieren und diese Stimmung zu erfassen. Ihm ist diese Seite, diese «exzentrische Bahn» immer übrig, das ist unbezweifelbar; aber, damit sie stattfindet, hängt diese, nach Hölderlin, von einer unerhörten Vereinigung des Begehrungsvermögen und der Fantasie unter der Führung der Freiheit $\mathrm{ab}$; d. h., es ist notwendig, dass eine Vereinigung, die in praktischer Hinsicht unfruchtbar ist oder wenigstens einen charakteristisch praktischen Umfang besitzt, jetzt theoretisch, d. h, nach einer Freiheit, durch die den von der Natur entgegensetzenden Widerstand wirklich fühlbar gemacht ist, betrachtet wird. Sonst, sagt Hölderlin, «würde es niemals einen vesten Zustand geben, der demjenigen gliche, der so eben angedeutet worden ist, wenigstens würde es nicht von uns abhängen, ihn vestzuhalten. Sein Gegenteil» fügt Hölderlin noch zu, «würde ebenso stattfinden, ohne dass wir es hindern könnten.» (TS: 3-4). Auch Hegel versuchte oft, in seinen frühen theologischen Texten dasselbe Problem auf dieselbe Weise, zu Kant zurückkehrend, zu lösen; und es ist deshalb nur eine Wende im Blick, die die Grundverschiedenheit zwischen den drei Freunden zeigt; denn was bei Schelling unstrittig und unfehlbar ist, was die Aufhebung der Antinomien betrifft, ist es nicht bei Hölderlin oder Hegel, die hier ihre eigene Autonomie innerhalb der Problematik erreichten und auf dieser Bahn immer bleiben würden. Ich zitiere deswegen Hölderlin, nur einige Monate später:

«In den philosophischen Briefen will ich das Prinzip finden, das mir die Trennungen, in denen wir denken und existieren, erklärt, das aber auch vermögend ist, den Widerstreit verschwinden zu machen, den Widerstreit zwischen dem Subjekt und dem Objekt, zwischen unserem Selbst und der Welt, ja auch zwischen Vernunft und Offenbarung theoretisch, in intellektualer Anschauung, ohne dass unsere praktische Vernunft zu Hilfe kommen müsste.» (SWB, 4: 229-230).

Vor allem aber - und hier, denke ich, ist der Kernpunkt unterscheidet sich Hölderlin nicht von Schelling nur dadurch, dass er eine neue Dimension des Problems darstellt. Er unterscheidet sich vielmehr, indem er dem deduktiven Prozess nicht eine ewige Bestätigung, nicht eine nuance von Unvermeidlichkeit, nicht einen prä-determinierten (kreisläufigen) Fortgang beilegt, sondern eine Stimmung, einen 
glückseligen oder einfältigen Augenblick und deswegen eine Dependenz des Subjekts von dem Objekt behauptet, der jene nicht nur hindert diese aufzuheben, sondern ihn auch dazu zwingt dieses als seinesgleichen zu betrachten. «Es ist ein bloßes Glück, so gestimmt zu sein» (TS: 3), sagt Hölderlin. Denn es handelt sich hier um einen einzigen Augenblick: ein Versöhnungsakt, der «himmlisch»(id.), unerreichbar, unfassbar, sogar unsagbar und unmenschlich ist, aber eben deswegen die einzig legitime im ewigen Fortschritt des Menschen zur eigenen Absolutheit war. So ist es auch ein Moment des Zweifels, ein Moment einer verschiedenen selteneren Produktivität, als die fichtesche, die von allen ihren Poren einen Duft des Unbekannten, sogar des Irrationalen, aushaucht. Die Beweisart des Problems, könnte man sagen, entblößt sich also von ihrer ehemaligen rationalen Umhüllung und nimmt die der scheinbaren Irrationalität als ihr wahrster Vorzug an; ein Gang der, so wie bei Schelling, ein Gang der Aufhebung der Persönlichkeit des Subjekts ist - denn so wie dort ist hier die Rede von dem Widererlangen eines Ursprungs, wo der Mensch als solches noch nicht existierte -, aber auch ein Gang, den man in umgekehrter Weise durchläuft, indem man sich eher von dem Strom des Schicksals, etwa teleologisch, bis zu seinem Zweck ziehen lässt, als das man nur dem gehorcht, das schon vorher bestimmt war.

Was bedeutete denn Hegels «legitimierte Idee von Gott» $(B, 1: 17)$ ? Hegels Antwort, von Hölderlin stark geprägt, scheint deutlich zu sein: eine Empfänglichkeit, die beweist, dass, indem man von der «Heiligkeit Gottes» (wie Fichte) ausgeht, eine Beweisart entsteht, die unrechtmäßig d. h., passiv, ohne die persönliche Teilnehmung des Subjekts - Gottes Bild vor dem Mensch herbeiruft, oder dass, wie bei Schelling, dies anfänglich legitim - dann aber absolut - aktiv wirkt, so dass es in keinem der Fälle eine wahre Rezeptivität, einen wahren Widerstand, als dem vom Selbst, gibt. Ganz im Gegenteil, behauptet Hegel, dass die Legitimität eher in uns selbst, durch unsere Sinnlichkeit erworben werden soll: ein Gefühl nach dem wir streben müssen, und das nur so empfangen werden kann; eine Empfänglichkeit, die nicht nur einnimmt, sondern auch zurückstößt, die nicht nur Zwecke empfängt, sondern ihren Sinn auch hervorbringt - und doch nicht wirklich hervorbringt, sondern zurückwirft, indem sie, anders als jene Empfänglichkeit, die erst fortfährt nachdem sie etwas Gegensätzliches erfährt, Zwecke empfängt. Denn hier nimmt die Zweckmäßigkeit das Aussehen eines umgekehrten Strebens an oder, wenn man will, eine neue Art Empfänglichkeit, ein süßer Ruf, den wir freiwillig verfolgen, dessen Ursprung wir aber nicht einsehen können. So wirkt, auf jeden Fall, der eigentliche doppelte Charakter des Strebens: ein Faktum, das sicher mit dem «sehr merkwürdigen Gedanken [in Ansehung der Antinomien]» $(B, 1: 20)$, die Hölderlin auch am 26.1.95 gegenüber Hegel erwähnt, die er aber nicht ausführte, verbunden ist. 
Auf diese Empfänglichkeit begründet sich letztendlich was Hegel unter einer «rein moralische[n] Natur» $(B, 1: 17)$ versteht. Sie ist also eine neue Art, die Welt anzuschauen, indem das Subjekt weder die Welt als seine eigene sieht, noch blindlings ihrem Ruf, der immer seine menschlichen Grenzen dem Objekt aussetzt, hinzueilt. Anders gesagt, der Mensch ist hier weder ganz aktiv noch ganz passiv, und also nicht antinomisch, weil der menschliche Gang, die Physikotheologie, eine energeia, ein lebendiges Gespräch zwischen den Gegenteilen ist; und deswegen ist eine «rein moralische Natur» jene, die, sich zwischen Gott und Mensch ausdehnend, beide so vereinigt, dass keine Vorränge existieren; sie ist eine vom Menschen als eine Seltenheit gefühlte Wechselseitigkeit, ein Augenblick der Irrationalität - und ich wiederhole - eine «Stimmung», die eine neue Ordnung der Dinge gebietet, oder wenigstens uns auf dem Gesichtspunkt dieser neuen Ordnung setzt. Nur dies erklärt, warum Hegels «legitimierte Idee von Gott» jetzt nur «rückwärts, in Erklärung der Zweckbeziehung» $(B, 1: 17)$ erreicht werden könnte, und warum diese Erklärung nichts als ein Augenblick ist, eine Stimmung von deren «Rezeptivität [es abhängt], wie in einem jedem einzelen die subjektive Religion beschaffen seyn soll» $(G W, 1: 88)$.

\section{RESUMO}

\section{O diálogo entre Hölderlin, Hegel e Schelling acerca das antinomias de Kant}

«Die Art, wie er den Mechanismus der Natur mit ihrer Zweckmäßigkeit vereiniget, scheint mir eigentlich den ganzen Geist seines Systems zu enthalten». Este passo, que dá origem ao presente ensaio, inscreve-se numa carta de Hölderlin a Hegel, conglomerando, todavia, as diferentes abordagens dos três amigos de Tübingen ao problema da filosofia da religião de Kant e à sua possível resolução, entre os anos de 1795 e 1796.

Sobressai do diálogo por estes estabelecido nas cartas uma leitura simultânea de Kant, suscitada pela nascente dissensão em relação à matriz ortodoxa do pensamento do Stift. O ponto de viragem - ou o ponto máximo - deste processo de afastamento dá-se, pois, justamente com a descoberta do «espírito do sistema» kantiano no que ele alia da explicação do fenómeno religioso ao do filosófico [«Die Art, wie er den Mechanismus der Natur mit ihrer Zweckmässigkeit vereiniget»]. Tal, creio, descobrem os três amigos faseadamente e não independentemente da formação do germe dos seus próprios sistemas de pensamento, no conceito da «Vorsehung», que já Kant, segundo Hölderlin, usara para «atenuar 
as suas antinomias», de que Hegel faz importante uso nos seus primeiros escritos religiosos e na formação da sua própria filosofia e que Schelling mais tarde exporá no seu Sistema do Idealismo Transcendental. Sobretudo, a providência impõe-se para os três como eixo da compreensão do homem perante a natureza e Deus e, assim sendo, como elo explicativo entre os pólos antinómicos que constituem a existência humana. Numa segunda instância, contudo - e é esta que pretendo enaltecer -, este momento decisivo para toda uma geração, para toda a história da filosofia, significa a consumação de uma nova perspectiva revolucionária originada em Kant, de uma nova visão do absoluto e do divino e, por conseguinte, de uma nova maneira de filosofar sobre a filosofia, menos filosófica do que até então, a ponto de a nova situação do homem e da sua reflexão humana no seio do problema o votarem ulteriormente, como parece acontecer heterogeneamente no pensamento dos três amigos, ao silêncio e à morte. O objectivo final deste ensaio passa, pois, por saber em que consiste este «letzte Schritt der Philosophie» e aquilo com que morre, o que tal passo terá representado e o que ele já então prenunciava para o desenvolvimento da filosofia.

\section{ABSTRACT \\ The dialogue between Hölderlin, Hegel and Schelling about Kant's antinomies}

«Die Art, wie er den Mechanismus der Natur mit ihrer Zweckmäßigkeit vereiniget, scheint mir eigentlich den ganzen Geist seines Systems zu enthalten»: This quotation, which originated the present essay, is solely extracted from a letter sent by Hölderlin to Hegel, and yet, it condensates three different approaches from the three Tübingen friends to the problem of Kant's philosophy of religion and to its possible resolution between 1795 and 1796.

From this epistolary dialogue emerges a simultaneous study of Kant, originated by the growing dissension towards the orthodox thought of the Stift. The turning point - or the maximum cumulative point - of this discordance happens precisely with the discovery of the «spirit of Kant's system», as a combined explanation of the religious and philosophical phenomena [«Die Art, wie er den Mechanismus der Natur mit ihrer Zweckmässigkeit vereiniget»]. This, I think, is something which the three friends discover gradually and not independently from the concept of «providence», which Kant himself, according to Hölderlin, had used to "attenuate his antinomies», which Hegel uses in his first religious writings and the initial formation of his own philosophy and which Schelling will later explore in his System of Transcendental Idealism. In a word, providence is consensually the comprehension axis between man, God and nature and, thus, the explanatory link between the antinomical poles which 
regulate human existence. On the other hand, however - this being the aspect I would like to stress -, this decisive moment for a whole generation, for the history of philosophy itself, means the consummation of a new revolutionary perspective born in Kant, a new vision of the absolute and the divine and, therefore, a new way to write philosophy about philosophy, less philosophical than before, to the extent that the new situation of man and his reflection within the problem ultimately destined them - as is the case in the three young philosophers - to silence and death. The final aim of this essay is, therefore, to know what this «last step of philosophy» is and what dies along with it, what such a step may have meant and what it already foretold in terms of the development of philosophy.

\section{Bibliographie}

Hegel, G. W. F. (1989), Gesammelte Werke, Bd. I. Frühe Schriften, Hrsg. Friedhelm Nicolin u. Gisela Schüler, Meiner, Hamburg.

Hegel, G. W. F. (1952), Briefe von und an Hegel, Bd. I (1785-1812), Hrsg. Johannes Hoffmeister, Akademie Verlag, Berlin.

Hölderlin, J. Ch. F. (1995), Sämtliche Werke und Briefe, Bd. 4. Briefe, Hrsg. Günther Mieth, Aufbau-Verlag, Berlin.

Hölderlin, J. Ch. F. (1998), Theoretische Schriften, Hrsg. Johann Kreuzer, Felix Meiner Verlag, Hamburg. 